\title{
La afectividad como recurso docente en el proceso de enseñanza-aprendizaje
}

\author{
Karla Paola Rengel Zambrano \\ Mercedes Naranjo Yépez \\ Ruth Loor Rivadeneira \\ Gabriela Narváez Olmedo
}

Universidad Técnica del Norte kprengel@utn.edu.ec

RESUMEN

La afectividad en el proceso de enseñanza-aprendizaje, es un factor indispensable para los estudiantes en su formación educativa debido a que aprenderín a gestionar sus emociones y las de los demás, devolvien formación educativa, debido a que aprendelan a gus percusión de la afectividad como recurso docente en el proceso de enseñanza-aprendizaje La metodologí usada tuvo un enfoque cualitativo con un diseño de estudio de caso en un grupo de estudiantes $y$ docentes a través de intros es trevista a estudintes. Los resultados revelaron que los docentes investicados encuesta a docentes y enla afectividad pero no la aplican, por ende, desconocen los efectos positivos que conlleva utilizarla como un recurso educativo efectivo y eficaz para el proceso de enseñanza-aprendizaje. Es así como los docentes requieren completar varias características para ser docentes afectivos encabezando desde la forma cono llegan a sus estudiantes a través de una actitud positiva, estrategias lúdicas e innovadoras de aprendizaje junto con la motivación constante dentro del aula. Finalmente se propone una gúa de estrategias para los docentes acerca de la afectividad como recurso docente.

Palabras clave: AFECTIVIDAD, PROCESO DE ENSEÑANZA, RECURSO, DOCENTE

\section{ABSTRACT}

Affectivity as a Teaching Resource in the Teaching-Learning Processy

Affectivity in the teaching-learning process is an indispensable factor for students in the educational process because they would learn to manage their emotions and those of others, giving teachers back their role as an example of life for their students. The objective of the study was to know the repercussion of affectivity as a teaching resource in the teaching-learning process. The methodology used had a qualitative approach with a case study design in a group of students and teachers through instruments elaborated by the author such as classroom observation, teacher survey and student interview. The results revealed that the investigated teachers know the importance of affectivity, but do not apply it, therefore, they are unaware of the positive effects of using it as an effective and efficient educational resource for the teaching-learning process. This is how teachers need to complete several characteristics to be affective teachers, leading from the way they reach their students through a positive attitude, playful and innovative learning strategies along with constant motivation within the classroom. Finally, a strategy guide is proposed for teachers about affectivity as a teaching resource. 


\section{Introducción}

En la actualidad el mundo ha cam biado constantemente, donde la sociedad y las necesidades de los alumnos han sufrido transformaciones, se ha descubierto que la afectividad es un factor decisivo en l proceso de enseñanza-aprendizaje y se busca que esto se implemente en las allas ce implemente en las aulas de clase, donde los docentes trabajen con un enfoque afectivo en todas las asignaturas y temáticas que se desean enseñar para que se pueda llegar a los estudiantes de manera positiva teniendo experiencia que les inviten a adquirir mayor motivación hacia el aprendizaje (Martínez Ambriz, 2018).

Desde la institucionalización de la escuela, la educación se ha vuelto un sistem rutinario basado en la misma metodología, ocasionando que el estudiante permanezca inactivo, sin interacción, con fatig y desanimo debido a que el docente no ocupa metodologías activas que generen empatía entre los actores del aprendizaje Córdova Huamán, 2017)

La sociedad ha cambiado vertiginosamente debido a los avances tecnológicos y a pesar de esto, se siguen ocupando métodos tradicionales de enseñanza. La mayoría de las dificultades que sobrellevan los escolares en las instituciones educativas, a más de la infraestructura, es la metodo- logía de enseñanza y el tipo de relaciones que se desarrolla entre estudiantes y docentes (López Aguilar, 2010).

A través de los años las institucione educativas han colocado en un espacio prioritario a lo cognitivo, caracterizado especialmente por la memorización los contenidos del currículo; es decir, no se pone énfasis en estimular los intereses $\mathrm{y}$ atender a las preocupaciones de los estudiantes. Varios docentes tienden a utilizar métodos arcaicos no solo para impartir conocimientos, sino, también para prefiere el uso de técnicas punitivas, ocupan calificativos (que pueden considerarse despectivos) para llamar la atención de sus pupilos y mostrar la desaprobación de la conducta que demuestran, se vuelven exigentes impartiendo instrucciones que se con ordenes rígidas, por ello, los docentes van colocando una barrera con sus estudiantes dando como resultado conductas no favorables en ellos (Pined Padilla, 2003).

La importancia del afecto y las emociones en el proceso de enseñanza- aprendizaje, se ha visto reflejada en el ser humano dependiendo de los estímulos que reciba, siendo estos sensaciones, emociones y estados de ánimo, abriendo las puerta a un filtro afectivo desencadenando estímulos positivos o negativos que dismi- nuirán sus límites en pro del ingreso de información o conocimiento nuevo, quedando registrado de manera más intens dependiendo de las emociones que surgieron en ese momento. Además del eje principal que es el afecto y las emociones, existen diferentes factores que nos permiten comprender de una mejor manera el aprendizaje, estos son los: 1) intrínsecos, 2) extrínsecos, 3) fuente del conocimiento y 4) método del educador (Arbaiza Meza 2018).

Es necesario que los docentes consideren que dentro del proceso de enseñanza-aprendizaje no solo se debe llega a los estudiantes con un contenido teórico, sino también que podrían apoyarse de un componente emocional, porque esto permitirá influir en el estudiante de manera positiva mediante las relaciones que establecen con ellos. Si los docentes mantienen un lazo afectivo con sus alumnos, generando entusiasmo y motivación, se podrá ocasionar cambios en el proceso de enseñanza-aprendizaje creando en lo estudiantes una actitud diferente que demuestre mayor confianza y emoción hacia el contenido que imparte el docente (Balderas, 1998).

Sería importante que los maestros, a ser un eje fundamental en la formación de sus estudiantes, no solo se centren en impartir contenidos, sino que, además, deen huellas significativas en el corazón de sus aprendices. El pensamiento martian de José Martí se basaba en una sola fórmula, en que el amor es el lazo para poder enseñar, que educar exige querer bie a los educandos, además menciona que la afectividad no debe generar temor y por lo tanto, hay que expresarla (Turner Marti \& Balbina, 2016).

La enseñanza afectiva tiene un impacto positivo sobre el crecimiento personal, intereses y experiencias en los estudiantes, así, la cognición y el afecto son esferas interactivas que trabajándolas conjuntamente tienen resultados positivos en el aprendizaje (García Cabrero, 2009).

Estudios aseguran que las caracterís- ticas de los docentes eficaces son: 1) preparación y organización de las clases, 2) claridad en la manera de impartir el contenido, 3) estimulación del interés de lo estudiantes junto con la motivación en el proceso de enseñanza -aprendizaje, mediante la manifestación de expresiones de entusiasmo, 4) relaciones positivas con lo alumnos, 5) demostrar altas expectativas y 6) mantener un clima positivo dentro del salón de clases basado en el respeto; de esta manera las caracteríticas de los numerales: 3, 4, 5 y 6 forman parte de las dimensiones afectivas en la enseñanza del profesor (Shechtman \& Leichtentritt, 2004;Shulman, 2006).

Las competencias afectivas en los do centes se basan en tres categorías: la autenticidad, respeto y la empatía; éstas a su vez ncluyen comportamientos de una persona genuina, entusiasta, consiente de misma y capaz de comportarse de acuerdo con sus sentimientos verdaderos; valora sus estudiantes con respeto, de forma positiva $y$ em persona que entiende sus sentimientos $y$ busca responder a sus necesidades. Esta competencias afectivas de los docentes tienen un impacto directo en el aprendizaje, pero sobre todo apoya, motiva y alienta al estudiante a explorarse así mismo a más de que brinda un aprendizaje significativo Olson \& Wyett, 2016).

Uno de los puntos más importante de la aplicación de la afectividad en el aula es la cercanía y la comunicación que incrementa la percepción física y psicológica de proximidad con los estudiantes correlacionándose de forma efectiva involucrando un aprendizaje cognitivo y afectivo agre gándose la motivación, el contacto visual lenguaje de inclusion, actitud distintiva entusiasta de los docentes, considerándose estos como atributos positivos (Godoy Zúñiga \& Campoverde Rojas, 2016).

La cercanía o inmediatez se demuestra a través de las conductas no verbale como gesticulación y expresiones de afabilidad, moverse alrededor de la clase interactuando con los estudiantes, so 
les, darles palmadas en el hombro, realizar choque de manos y vocalizaciones adecuadas (Ginsberg, 2007).

Finalmente, otro de los aportes de la enseñanza afectiva en las aulas es que genera en los estudiantes altos niveles de autoconcepto, ganancias a nivel intelectual $y$ creatividad, resolución de problemas, mayor participación y menos problemas de disciplina, impactando en la adquisición de habilidades académicas, personales y sociales (Olson \& Wyett, 2016).

La presente investigación se enfoca en el estudio de la afectividad como recurso docente en el proceso de enseñanza- aprendizaje de los estudiantes de Escuela de Educación Básica Particular Florentinos, con el propósito de guiar a lo docentes de la institución en la aplicación de una enseñanza más afectiva repercutiendo positivamente en su eficiencia metodológica. Además, como objetivos específicos se planteó, describir la afectividad que emplean los docentes para un clima adecuado con los estudiantes; compara las percepciones sobre la afectividad entre docentes y estudiantes y diseñar estrategias de afectividad para el proceso de enseñanza- aprendizaje.

\section{Metodología}

En esta investigación se empleó una metodología con enfoque cualitativo, orientado en el estudio de las variables de la afectividad en el proceso de enseñanza - aprendizaje en la cátedra en la Escuela Particular "Florentinos", de la ciudad de Quito. Se consideró tres criterios de anáisis acerca de la afectividad: la motivación por parte del profesor, la inteligencia interpersonal del maestro y la sensibilidad de educador con la finalidad de elaborar una guía de diseño de estrategias que aporten a la institución educativa.

El análisis de la investigación se llevó a cabo mediante la revisión bibliográfica, donde se explica el valor de la afectividad como un requisito primordial en el proceso enseñanza-aprendizaje, y se expone las consecuencias positivas de implementar una educación afectiva. Adicionalmente se analizaron las respuestas obtenidas de grupo de participantes seleccionados con antelación, buscando conocer el contexto real de la institución educativa relacionada la afectividad en el aula, la motivación de los estudiantes y la actitud del docent frente a sus alumnos.

El proceso metodológico que se siguió consistió en definir el concepto de afectividad, las vertientes que la caracterizan y a importancia de su implementación en el salón de clase. Analizar y extraer las ideas esenciales, las perspectivas de la población de estudio y por último sintetizar la información obtenida de la observación áulica pertenecientes al grupo de estudio.

La investigación toma como base la Declaración de Helsinki de la Asociació Médica Mundial (2013) y los Principios Éticos de los Psicólogos y Código de Conducta de la American Psychological Association APA (2010), los cuales tienen el fin de velar por el bienestar y los derechos de confidencialidad de los actores a participar en la investigación respetando su integridad, estándares éticos, legales, jurídicos y bioéticos que se han tomado en cuenta con el objetivo de generar conocimientos siempre guardando la confidencialidad de las personas participantes.

\section{Participantes}

Para este estudio, los participantes seleccionados de manera no probabilística fueron 8 estudiantes que cursan el séptimo año de educación general básica de la Escuela de educación básica Particular "Florentinos" de la ciudad de Quito, Ecuador de los cuales 4 fueron del género femenino y 4 del masculino de edades comprendidas entre 11 y 12 años, quienes han permanecido en la institución educativa desde temprana edad, dándonos a conocer una perspectiva global de la institución educativa con los docentes.

También se contó con la participación de 5 docentes que laboran en la institución desde un tiempo considerable, siendo as que 3 de ellos han trabajado durante tres años y los dos restantes 1 año 5 meses, po ello, los estudiantes conocen las actitudes de los docentes por las interacciones concurrentes dentro del salón de clase y durante las horas de receso.

\section{Instrumentos}

Para la investigación se utilizaron tres instrumentos de elaboración propia, que fueron validados por tres expertos en el campo profesional. Estos instrumentos tienen la finalidad de recopilar información relevante sobre la afectividad como recurso utilizado por los docentes en el proceso de enseñanza aprendizaje desde la perspectiva de los maestros y estudiantes.

El primer instrumento se basa en una observación áulica virtual a través de la plataforma zoom realizada con los docentes y estudiantes, la cual contiene 11 preguntas sobre la relación del maestro hacía los estudiantes y la metodología de enseñanza, con opciones de respuesta de siempre, casi siempre, a veces y nunca. segundo instrumento es una entrevista abierta realizada a 8 estudiantes a través de la plataforma zoom la cual consta de 15 preguntas elaboradas con el fin de recabar información necesaria sobre la percepción de los alumnos sobre los docentes en cuanto a su relación y metodología de enseñanza. Finalmente, el tercer instrumento se basa en una encuesta que fue aplicada a los docentes mediante una coevaluación con el fin de obtener datos relevantes sobre las competencias y características afectivas que presentan durante la interacción con sus estudiantes.

\section{Procedimiento}

Se efectuó una visita a la institución educativa seleccionada en la que se dio conocer a las autoridades sobre la investigación a realizar con los estudiantes y do- centes, solicitando una autorización previa para la ejecución del estudio para conoce el tema, objetivos y un diseño de estrategias para los docentes según los datos recabados.

Con los docentes participantes se realizaron cinco sesiones:

- En la primera sesión, se elaboró una reunión con los docentes que imparten clases a séptimo de educación básica general donde se expuso el tema, objetivos, fin y el beneficio de la investigación para la escuela, se solventaron dudas y se suscribió el consentimiento informado en el cual se solicitaba la participación voluntaria en la investigación a realizar.

- En la segunda sesión, la cual fue de manera virtual, se llenó la ficha sociodemográfica obteniendo datos relevantes en relación al trabajo de los docentes y e tiempo que laboran en la institución educativa.

- Durante la tercera sesión, se realizó la observación áulica a las clases virtuales de los docentes participantes donde se recabó información sobre la metodología de enseñanza y la relación con los estudiantes, ítems que son parte de los indicadores de la investigación.

- En la cuarta sesión, se aplicó una encuesta a los docentes obteniendo información sobre datos relevantes en cuanto al tema de investigación.

- En la quinta y última sesión, se realizó una reunión con los docentes y autoridades de la institución educativa para darles a conocer los resultados obtenidos que permitan emitir recomendaciones una guía de estrategias enfocadas a la afectividad.

Con los estudiantes se realizaron 3 sesiones:

- En la primera, se les informó a los padres de familia y alumnos sobre el tema de investigación, objetivos y su rol en el estudio y adicional se solicitó la autorización por parte de los representantes mediante un consentimiento informado y un asentimiento por parte de los estudiantes.

- En la segunda sesión, se aplicó la 
ficha sociodemográfica de la familia en general en la que se recabó información sobre los participantes.

En la tercera y última sesión, se realizó una entrevista a los participantes través de la plataforma zoom con preguntas abiertas en relación al tema de investigación, adicional se dio lectura y se les hizo firmar un consentimiento informado de confidencialidad y de participación voluntaria, por lo cual se le informó que la grabación realizada seria confidencial y con fines investigativos y que ninguna persona, a más de la investigadora tendría acceso a ella.

\section{Resultados}

Se presentan a continuación los resultados específicos recopilados con cada instrumento.

\section{Observación Áulica}

En la observación áulica, las actitudes de los docentes que mantienen durante la clase con los estudiantes reflejaron los siguientes resultados como se indica en la Tabla 1.

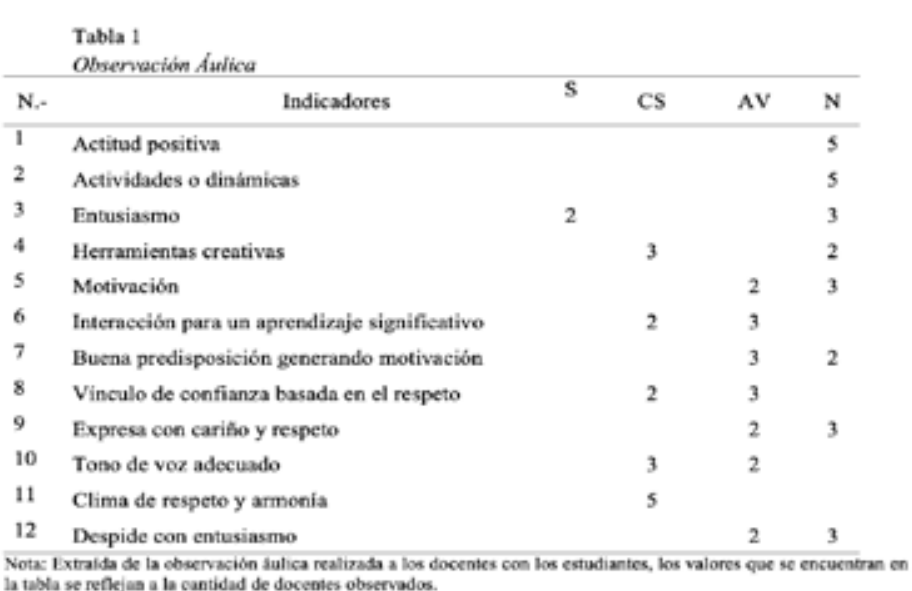

Los resultados que fueron obtenidos dentro de los indicadores como la actitud positiva del docente (comportamientos afables que muestran e influyen en la instrucción de conocimientos) y las ac tividades o dinámicas al inicio de clases, son las más preocupantes ya que no se evidenciaron durante la observación áuli- ca, mientras que la motivación se aplica en una escala de a veces o nunca, lo cual resulta un tema debatible siendo este un indicador muy relevante que forma parte de las características afectivas. Se puede resaltar en los docentes el respeto que casi siempre y a veces se pudo observar con los estudiantes, los docentes al expresarse con cariño a veces y nunca se mantienen serios durante toda la clase y se dirigen con respeto al llamar a un estudiante, tono de voz es adecuado en las escalas de casi siempre y a veces, y es utilizado para controlar la disciplina de los estudiantes y por último el entusiasmo (comportamiento no verbal, que contribuye a la eficacia que mantiene el interés de los estudiantes evitando desgano y falta de atención, favoreciendo a su aprendizaje) se lo denota en dos docentes que tienen afinidad hacia los padres de familia y estudiantes.

\section{Encuesta a docentes}

En la encuesta a docentes se obtuvieron datos relevantes sobre las competencias y características afectivas que presentan desde una coevaluación a sus compañeros.

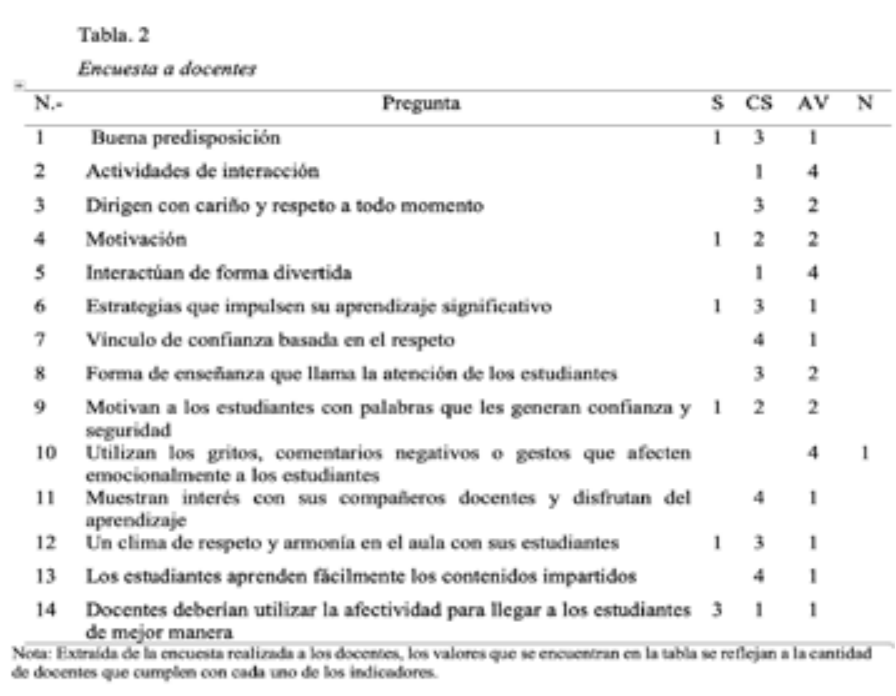

Los resultados que fueron obtenidos dentro de la encuesta a docentes en relación con la interacción, la predisposición (actitud mental adecuada para un determinado propósito o actividad) y el interés al impartir las clases con sus estudiantes los docentes lo realizan en escalas de a veces y casi siempre, con una similitud de respuestas contestadas por cada uno de los participantes. Cabe recalcar que los docentes tienen una percepción más amplia en sus respuestas, en comparación con la observación áulica, porque también se refieren al tiempo de clases de manera presencial, dando una perspectiva que están cerca de cumplir con las características necesarias para ser docentes afectivos. Dentro del indicador de motivación los resultados son similares, los docentes perciben a un compañero que motiva los estudiantes todo el tiempo, mientras que los demás docentes se mantienen en escalas de casi siempre y a veces, esto indica que los docentes motivan a sus estudiantes, pero no de una forma constante. Otro de los resultados en base a las formas de enseñanza- aprendizaje, muestra que los docentes en su mayoría cas siempre y a veces se mantienen en una forma de enseñanza que causa interés en los estudiantes, pero no conllevan que sea tan significativo si utilizan una forma de enseñanza rutinaria para que los estudiantes aprendan fácilmente los contenido impartidos. En cuanto a la afectividad se puede mencionar que los docentes ven importante el uso de la afectividad en su mayoría para que el aprendizaje lle gue hacia sus estudiantes. Sin embargo, las características afectivas como el dirigirse de forma cariñosa, se mantienen en escalas de casi siempre y a veces, lo que evidencia que aun los docentes necesitan interiorizar sobre la importancia de ser un docente eficaz y afectivo, ya que un de las cosas preocupantes que se tuvo en esta encuesta es que los docentes afirman que sus compañeros utilizan gritos o comentarios que afectan emocionalmente los estudiantes y se resalta que el docente que fue mayormente mencionado con características positivas, nunca se expres de esta manera hacia sus estudiantes.

\section{Entrevista a estudiantes}

En la entrevista a estudiantes se valor las respuestas en relación a la percepción que tienen sobre sus docentes, para ello se elaboró un instrumento con 7 indicadores dentro de los cuales se encuentran: 1) Car acterísticas afectivas, 2) Confianza, 3) Motivación 4) actitud, 5) clima de aula,6) Metodología, y 7) afectividad.

Se hace un compendio de todas las respuestas obtenidas y estos son los resultados.

En cuanto a las características afectivas que los estudiantes participantes coinciden de forma reiterativa de sus docentes es que sean calmados, dinámicos, amigables, carismáticos, motivadores, inspiradores de confianza, divertidos, con actitud positiva, cariñosos, amables, sin embargo hay estudiantes que mencionan ciertas características más relevantes en un docent "[...] me gustaría que los docentes domin en los temas que enseñan y que las clases que se imparten las realicen con dinámicas sin monotonías". Finalmente se agrega que los docentes deben siempre contar con material de apoyo, "[...] los docentes de ben utilizar ejemplos, juegos, además de ser pacientes y amables porque así se les puede entender mejor y así poder preguntarles con confianza".

En relación con la confianza, los estudiantes mencionan, "con algunos docentes no, porque a veces da vergüenza pre guntar y porque son muy serios", siendo la confianza parte de los factores de la afectividad los estudiantes mantienen comentarios sobre la seriedad de sus docentes, "[...] les falta ser más amigables y que no sean de carácter fuerte", sin embargo, hay docente que son nombrados de forma concurrente con buenos comentarios por parte de sus estudiantes, "[...] me generan confianza porque cuando no entiendo algo ellos me pueden explicar y sacarme la duda de que es esto o aquello", "[...] Si, porque si tengo algún inconveniente o problemas para en tender me ayudan explicando nuevamente".

La motivación de los docentes hacia los estudiantes se ve reflejada, no obstan- 
te, no todos la realizan. Los estudiantes mencionan que algunos docentes los motivan con frases como[...] "tú puedes", "si te propones lo lograras", en cambio otro docentes no les mencionan frases motivadoras, utilizan palabras como "[.. no hace nada en la casa para que no traiga las tareas", "[...] ya voy a llamar a su mama, para que hable con usted y saber qué le pasa", "[...] si no me entiende debe acercarse con tiempo" o "[...] debe estudiar si no se va a quedar de año", también hay docentes que se muestran indiferentes como menciona un estudiante "[...] no $\mathrm{m}$ dicen nada solo imparten su clase y ya". También se menciona que cuando ellos se sienten tristes o con poca motivación en sus actividades escolares algunos docentes no les dicen nada pero otros si emiten comentarios positivos, los hacen reír o los animan, como el comentario que emitio un estudiante sobre un docente "[...] me hace participar para que cambie de actitud $y$ este feliz".

Por otro lado, el indicador de actitud de los docentes en su mayoría los estudiantes están de acuerdo que son serios, ex gentes, respetuosos, poco pacientes y comprensibles, algunos amables y otros indiferentes para entablar una conversación. Se puede ver que la forma como el docente pus trata os trata puede influir en la forma de aprender " $[. .$.$] los docentes tienen un tono$ de voz un poco fuerte que a veces da miedo me gustaría que sean más calmados y antes de iniciar la clase haga una dinámica" otro estudiante menciona "[...] El cariño y la amabilidad pueden influir en que yo tenga confianza en ellos en las clases para yo decirles cualquier duda que yo tenga".

En relación al clima de aula concuerdan que existe una sola docente que cumple con esta característica de forma afectiva, a la que describen "[...] Hace dinámicas, ejemplos y videos interactivos además de que me siento en confianza para poder preguntarle algo que no entiendo"; el resto de docentes generan un clima de respeto en el aula pero son muy serios, "[...] si tienen al aula o a la clase en respeto, pero no no

\section{tratan con cariño"}

Dentro de los indicadores se toma en cuenta el proceso de enseñanza - aprendizaje, los docentes consiguen con los estudiantes un aprendizaje significativo a través de estrategias innovadoras, los comentarios más relevantes son " $[. .] M$. gusta como enseñan algunos porque realizan juegos y nos saca al patio para poder aprender mejor", por otra parte un estudiante menciona de manera afirmativa "[ in con una actitud positiva para aprender", tenemos comentarios negativos "[...] No, porque algunos profesore no hacen dinámicas y no hacen las clases divertidas" ; al mencionar si los docentes participan y utilizan estrategias innovadoras y divertidas los estudiantes mencionan que los docentes no participan con ellos, los comentarios más relevantes obtenidos por parte de los estudiantes "[...] Algunos porque otros solo decían saquen el libro comenzaban a dar la clase", otra respuesta fue "[...] No todos, porque a veces están sin ánimo" y algunos estudiantes mencionan de manera positiva a un docente "[...] si porque parece raro pero no, con los juegos también puedes aprender ejemplo: pueden jugar a la rayuela y puedes ir aprendiendo los números en inglés, etc.".

La afectividad para los estudiantes en su totalidad respondieron que si es importante que el docente sea afectivo con ellos resaltando los siguientes comentarios, "[... Si porque no nos da miedo y es divertida su clase", "[...] Si porque ayuda a que los estudiantes les tengan confianza." [...] "Si una manera respetuosa, porque así, también es una forma de motivar a los estudiantes", de manera general para los estudiantes es importante que los docentes sean afectivos porque así se abre las puertas de la confianza y de esa manera el aprendizaje alcanzaría los resultados esperados.

\section{Discusión}

De acuerdo con diferentes investigaciones se puede concluir que la afectividad de los docentes hacia los estudiantes causa resultados positivos dentro del aula de clase tanto en la disciplina como en e proceso de enseñanza - aprendizaje. S recalca la importancia de la afectividad dentro del aula, ya que los estudiantes se muestran más participativos dentro de ell (García Rangel \& García Rangel, 2014) Los estudiantes que participaron en est investigación reafirman esta realidad a nombrar constantemente al docente que resalta entre los demás por cumplir con la mayoría de características y aspectos necesarios para ser un docente eficaz y afectivo, lo que provoca una mayor predisposición por parte de sus alumnos.

Se menciona que las instituciones educativas colocan de forma prioritaria a cognitivo, de esta manera se puede hace un análisis en que la institución educativa busca ser la mejor dentro del sector en el cual se encuentra ubicado, por ende lo h logrado debido a la demanda de estudiantes que han tenido por sus buenas referencias en cuanto a su oferta educativa sin embargo, aún se necesita implementa dentro de la institución educativa la parte afectiva junto con metodologías activa que lleven a los estudiantes a tener mayo interés, agregando en los docentes diferentes estrategias relacionadas a la importancia de la afectividad para evitar crear una barrera que generen en los educandos vergüenza, miedo, desinterés (Pineda $\mathrm{Pa}$ dilla, 2003). Si los docentes mantienen un lazo afectivo con sus alumnos, generando entusiasmo y motivación, se podrá ocasionar cambios en el proceso de enseñanza-aprendizaje fomentando en ellos un actitud diferente que denote confianza emoción hacia el contenido que imparte el docente

En la investigación de Godoy Zúñiga \& Campoverde Rojas(2016) realizada en diferentes países como Argentina, Colombia y Ecuador hace mención en su investigación un apartado "(...) La comunidad educativa ecuatoriana posee muchas gana por entender a su alumnado y convertirse en mostrar modelos de enseñanza, (...) no existe un pensamiento unificado para la elaboración de proyectos con estrategias pedagógicas que coadyuven al acercamiento entre docentes y los adolescentes o jóvenes (...)" (p11). Con esto se ve la necesidad imperante de que los docentes sean entes afectivos dentro del salón de clase mejorando de esta manera el nivel académico y emocional que conllevan a un desarrollo integral del estudiante, este estudio se de bería realizar a nivel de todo el sistema educativo teniendo en cuenta estos aspectos importantes para solventar el acercamiento afectivo entre docentes y estudiantes.

Dentro de las limitaciones que se pudieron, palpar en la investigación realizad fue el estado de emergencia por el COVID 19 y la subjetividad del instrumento de observación áulica que se lo realizo para efectuarla de manera presencial, se sugiere que para obtener resultados más fiables se podría realizar de manera presencial y de forma concurrente por un mes, agregando dentro de la metodología un enfoque cualitativo y cuantitativo y para un mayo número de estudiantes. Además, hacer una comparación entre el docente que tiene más competencias afectivas al que no la tiene y hacer un análisis con el rendimien to académico, de esta manera se tendría información más relevante acerca de cómo influye la afectividad en los estudiantes.

\section{Conclusiones}

La afectividad dentro del proceso de enseñanza-aprendizaje tiene una influencia positiva, el docente al ser afectivo mostrando gestos, utilizando un tono de voz adecuado, siendo cariñoso, empático entusiasta con sus alumnos permite que ellos aprendan de manera significativa, de tal manera que los estudiantes al ver esta características generan confianza hacia maestro; sin embargo, hay docentes que se muestran distantes durante las clases causando vergüenza, miedo, nervios, falta de confianza y desmotivación lo que impide tener un proceso de enseñanza aprendizaje adecuado.

Los docentes cumplen con ciertos 
aspectos importantes dentro de la afectividad, no obstante, necesitan conocer su importancia dentro del proceso de apren dizaje para mejorar el clima del aula; dentro de los aspectos que necesitan mejorar se encuentra la motivación, la generación de confianza, entusiasmo y el desconocimiento de metodologías activas y lúdica que brindarían resultados más positivos al momento de enseñar.

Para concluir, tanto maestros como estudiantes consideran que la afectividad como recurso docente es relevante dentro del proceso de aprendizaje, a pesar de ello no todos los maestros, aún sabiendo la importancia de aplicar una metodología acti- va, afectiva y eficaz, la implementan en sus clases debido a la falta de conocimiento de estrategias en este ámbito.

\section{Anexos}

\section{Objetivo}

Sensibilizar a los docentes en la importancia de la afectividad dentro del proceso de enseñanza-aprendizaje.

\section{Destinatarios}

Todos los docentes pertenecientes a la institución educativa $\cdots$

\begin{tabular}{|c|c|c|}
\hline ise & Contenidos & Tiempo \\
\hline Sensit & ocente & in \\
\hline plan & Caracteristicas de un docente afectivo y eficaz & $15 \min$ \\
\hline $\begin{array}{l}\text { capacitación } \\
\text { docentes }\end{array}$ & $\begin{array}{l}\text { La importancia de crear un clima de aula basado en la } \\
\text { afectividad }\end{array}$ & $30 \mathrm{~min}$ \\
\hline empo & 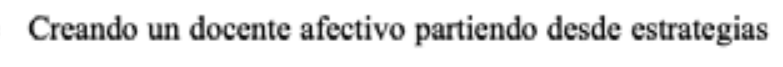 & \\
\hline
\end{tabular}

ejecución en 3 innovadoras mediante la practica

Revisión de manual de estrategias con técnicas aplicadas $45 \mathrm{~min}$

a mejorar el clima de aula, confianza, respeto basado en

el cariño, motivación, tono de voz, metodología innovadora.

Evaluación y Se realizará visitas áulicas y se corroborará lo aprendido

\section{seguimiento en la capacitación.}

Tiempo de Se guiará a los docentes en caso de necesitarlo, $30 \mathrm{~min}$

ejecución en 5 retroalimentándolo en sus aplicaciones.

dias

\section{Referencias bibliográficas}

Alonso, Balmori, Coon, Mitter, \& Cosacov. (2010). Lectur de la afectividad. Mexico: Repositorio de sitios experimentales psicologia Unam.

Arbaiza Meza, C. (27 de Julio de 2018). Centro peruan de audición, lenguaje y aprendizaje . Obtenido de CPAL: https://cpal.edu.pe/novedad/la-importancia-del-afecto-y-las-emociones-en-el-proceso-deaprendizaje/

Balderas, C. (1998). Influencia de la personalidad, intelgencia y afectividad en el rendimiento escolar. (F. Pedagogi, Ed.) Veracruz: Universidad Veracruzana.

Córdova Huamán, E. M. (2017). Enseñanza afectiva y proceso de aprendizaje. Peru: Universidad César Vallejo. Obtenido de https://repositorio.ucv edu.pe/bitstream/handle/20.500.1269

De la Caba, M. (2019). Educacion Afectiva. Universidad de Caba, M. (2019). Educacion Afectiva. Universidad de
País Vasco. Obtenido de http://www.sc.ehu.es/miw-

García Cabrero, B. (01 de Noviembre de 2009). Las dimensiones afectivas de la docencia. Revista Digital Un versitaria, 10(11),2-14. Recuperado el 01 de Diciembre de 2020, de http://4mw

García Rangel, G. E., \& García Rangel, A. K. (2014). RELACIÓN MAESTRO ALUMNO Y SUS IMPLICACIONES EN EL APRENDIZAJE. Ra Ximhai, vo 10( núm. 5), 10(5),279-290. Recuperado el $29 \mathrm{~d}$ Noviembre de 2020, de https://www.redalyc.org/ar$\mathrm{d}=461 / 46132134019$

Garritz Ruiz, A. (2009). La afectividad en la enseñanza de la ciencia. (E. química, Ed.) Scielo, 20(1), 212219. Obtenido de http://www.scielo.org.mx/scielo php?script=sci_arttext\&pid=S0187-893X200900050 $0002 \&$ lng $=e s \&$ tng $=e$

Ginsberg, S. (2007). Teacher transparency: What students can see from Faculty communicatio nido de htp//wwwjcalemoryed//viea Obephp?id=84\&layout=html

Godoy Zúnigas M. E \& Campoverde Rojas, B. J. (2016). Análisis comparativo sobre la afectividad como motivadora del proceso enseñonza-aprendizaje casos: Arsentina Colombia y Ecuador Sophia (2) 12(2) 217.231. doi http//dxdoiong/10.1863/
González,A., \& González, M. C. (2000). La afectividad en el aula de clase. Colombia Médica, 31(1), 55-57. Recuperado el 29 de Noviembre de 2020, de https://www. redalyc.org/articulo.oa?id=283/28331111

Lescano, C., \& Natalia, B. (19 de Junio de 2015). El Mielnio Aprendizaje y Afectividad. Obtenido de https://lmilenio.info/2015/06/19/aprendizaje-y-afectividad/

López Aguilar, N. G. (2010). EL ABURRIMIENTO EN CLASES. Procesos Psicológicos y Sociales, 6(1 y 2), 1-43. Recuperado el 01 de 12 de 2020, de https:// www.uv.mx/psicologia/files/2013/06/El-Aburri-

miento-En-Clases.pdf
Martínez Ambriz, M. (05 de 08 de 2018). Del pensar al sentir:A IMPORTANCIA DE LA AFECTIVIDAD EN EL PROCESO DE ENSENANZA-APRENDIZAJE. Correo del Maestro, 1-4. Recuperado el 01 de Diciembre de 2020, de https://www.correodelmaestro.com/publico/html5082018/capitulo5/del_pensar_al_sentirhtml

Olson, C. O., \& Wyett, J. L. (2016). Teachers need affective competencies. Education, 120. Recuperado el 01 de Diciembre de 2020, de https://www.questia.com/ tive-comarnor tive-competencies

(iz Ocaña, A. (2013). PEDAGOGÍA DEL AMOR Y LA FetCiDAD. Bogotá: Ediciones de la U. Recuperado 20132369011/Libro_Pedagog/C3\% ADa_de Amor_y_la_Felicidad

Pineda Padilla, R. (2003). La importancia de la afectividad en educacion primaria. Mazatlán, Sinaloo http:/20023.11351/pdf/23604 $283.51 / 23$

Shechtman, Z., \& Leichtentritt,J. (2004)...Affective teaching pean Journal of Teacher Eduction , 27(3) $323-333$ doi.https//doiorg/101080/0261976042000290822

Shulman L. (2006). Ways of seeing ways of knowing ways of teaching ways of learning about teachin. Journ of Curriculum Studies, 23, 393-395, doint tourns org/10.1080/0022027910230501

Turner Marti, L., \& Balbina, P.C. (2016). Pedagogia de la ternura. (E. Matreros, Ed.) Biblioteca popular los libros bre de 2020, de https//issu com/lbibliopop/docs marti-pedagogia-de-la-ternura 